\title{
INFRARED IMAGING DEVICES IN INFRARED MEDICAL RADIOGRAPHY
}

\author{
William L. Wolfe \\ Institute of Science and Technology, The University of Michigan \\ Ann Arbor, Mich.
}

The word "thermograph" often used to describe both an instrument and the product of that instrument has as its roots the Greek words thermos and graphos. The latter means a sketch or pictorial representation; thus, the graphic arts, scientific graphs, photographs, etc. The former relates to heat or temperature. At first reading, then, the word "thermograph" is most appropriate for a graphical representation of the heat or temperature distribution of any body. But an infrared instrument does not measure either of these; it measures the distribution of the infrared radiation (more properly, radiant emittance or radiance). Thus a more appropriate and precise name for the instrument is an infrared radiograph. By analogy the photographic output could be called an infrared radiogram, and the technique, infrared radiography. This paper describes some of the instrument design principles.

\section{Instrument Design}

The success of infrared radiography depends in large measure upon the degree to which physicians are able to interpret the results and relate them to diseases and how well the engineers are able to design adequate instruments. Such instruments are based upon the application of simple concepts of optics and electronics -sometimes requiring ingenious solutions to special problems. Design concepts and compromises are discussed in this section; basic radiometric theory is presented in Appendix $A$.

Two quantities seem to be the principal desiderata: the smallest determinable temperature difference and the time necessary to scan a given portion of the body with a specified resolution. Every electro-optical system is limited by noise (like the static of a radio, caused by such things as the random emission of electrons from an electron-tube cathode or the Brownian motion of electrons flowing through a resistor as they are jostled by its crystal lattice) so that the minimum detectable temperature difference can be defined as temperature difference that gives a signal just equal to the average noise signal; this is usually called the Noise Equivalent Temperature Difference (NETD). Nothing in nature responds to change immediately. Some things respond relatively slowly; infrared detectors respond fairly quickly. The time it takes for a detector to give an output 63 per cent of what it will eventually give is called the time constant-in two time constants it gives about 95 per cent. Thus a detector must be permitted to "view" a spot for about two time constants $(2 \tau)$ or its output will be seriously degraded.

The equations from which much can be inferred about equipment designs and compromises is derived in Appendix B; it is the following:

$$
\begin{aligned}
& \text { NETD }=\left(\frac{\pi}{\sigma}\right)\left(\frac{1}{\epsilon \mathrm{T}^{3}}\right)\left(\frac{\Omega}{\omega^{2}}\right)\left(\frac{1}{\eta \overline{\mathrm{A}_{\mathrm{c}}}}\right)\left(\frac{\sqrt{\mathrm{A}_{\mathrm{d}}}}{\mathrm{D}^{*}}\right)\left(\frac{1}{\mathrm{t}}\right) \\
& \pi=3.14 \\
& \sigma=5.667 \times 10^{-12} \\
& \epsilon=\text { radiating emission efficiency of the human body } \\
& \mathrm{T}=\text { body temperature }
\end{aligned}
$$




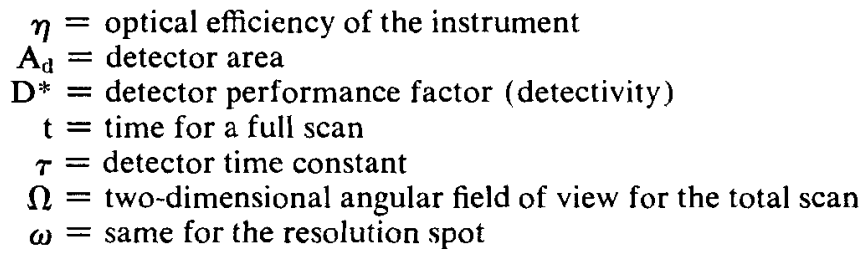

This form of the equation for the minimum detectable or noise equivalent temperature difference emphasizes the fact that NETD is dependent upon the properties of the patient, the patient-instrument geometry, the optical system and the detector. The terms within the first parentheses are just constants. The terms in the second parentheses relate to how much the patient radiates. If he has a high emissivity, as most people do $(\epsilon \approx \approx 1)$, and relatively high temperature, then smaller temperature differences can be detected. Of course $\epsilon$ and $T$ for humans are relatively constant, neither changing by more than a few per cent. Thus this is not really subject to engineering modification in equipment design. The terms within the next parentheses indicate that the smaller the total field and larger the resolution field, the smaller the NETD will be. Thus to get better temperature discrimination, the instrument should scan a smaller portion of the body and do it in larger steps. These are contrary to what is usually desired. The terms within the fourth set of parentheses indicate that an efficient optical system with a large collector should be used. The next group of terms indicates a sensitive detector with a small area should be used. Finally the scan time should be long. As nature would have it, $\Omega, \omega$, and $t$ all enter the equation the wrong way. For reasons of convenience, ease of interpretation, and repeatability $\Omega$ should be large while $\omega$ and $t$ should be small. For smaller detectable temperature differences it should be just the opposite - and here is the difficulty.

This equation is fairly easy to use. Values for $\sigma$ and $\pi$ are already given; $\epsilon$ is about one and $\mathrm{T}$ about $300^{\circ} \mathrm{K} ; \Omega$ is the total scanned area divided by the square of the instrument-to-subject distance; $\omega$ is the resolution spot area divided by the square of the instrument-subject distance; $\eta$ is about $0.5 ; \mathrm{A}_{\mathbf{c}}$ can be varied from about five $\mathrm{cm}$. to $30 \mathrm{~cm} ; A_{\mathrm{ll}}$ is about one $\mathrm{mm} .^{2} ; t$ is chosen; and values of $\mathrm{D}^{*}$ are given in FIGURE 1 .

FIgURE 1 shows the $D^{*}$ curves for a wide variety of detectors and TABLE 1 gives additional data. FIGURE 2 shows the transmission regions of optical materials which may be useful. Most of these materials are unsatisfactory for one reason or another so that mirrors are generally used. Mirrors for infrared use can be very efficient in that materials with very high reflectivity are available and good designs for narrow fields are relatively easily made. Some of the solutions, illustrating different design techniques are discussed below. Clearly these illustrations do not exhaust the techniques.

To some extent matters have been simplified in order to present the equations in such a concise form. The actual design is based on these but also on experience. Infrared measurements are among the most difficult in all of physics and require ingenious and meticulous attention as well as the straightforward application of the known principles of optics and electronics. How does one make sure that calibration standards are constant and accurate, that stray radiation and reflections do not obscure the values of the precise measurements being made? Should lenses with their problems of materials, or mirrors with their problems of obscuration be used? All these questions must be left to the designer. Only some solutions from the many possibilities can be enumerated here. 


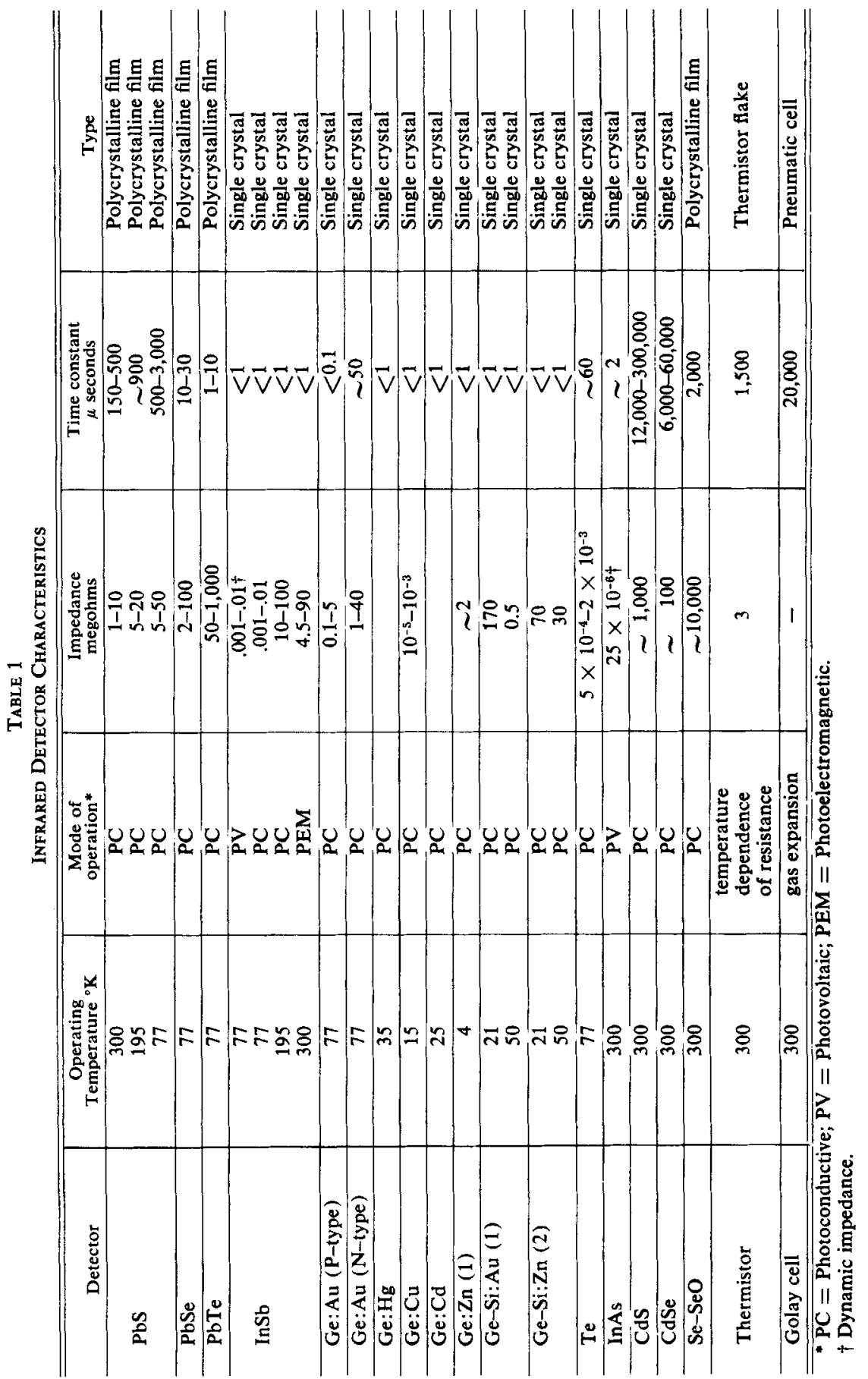




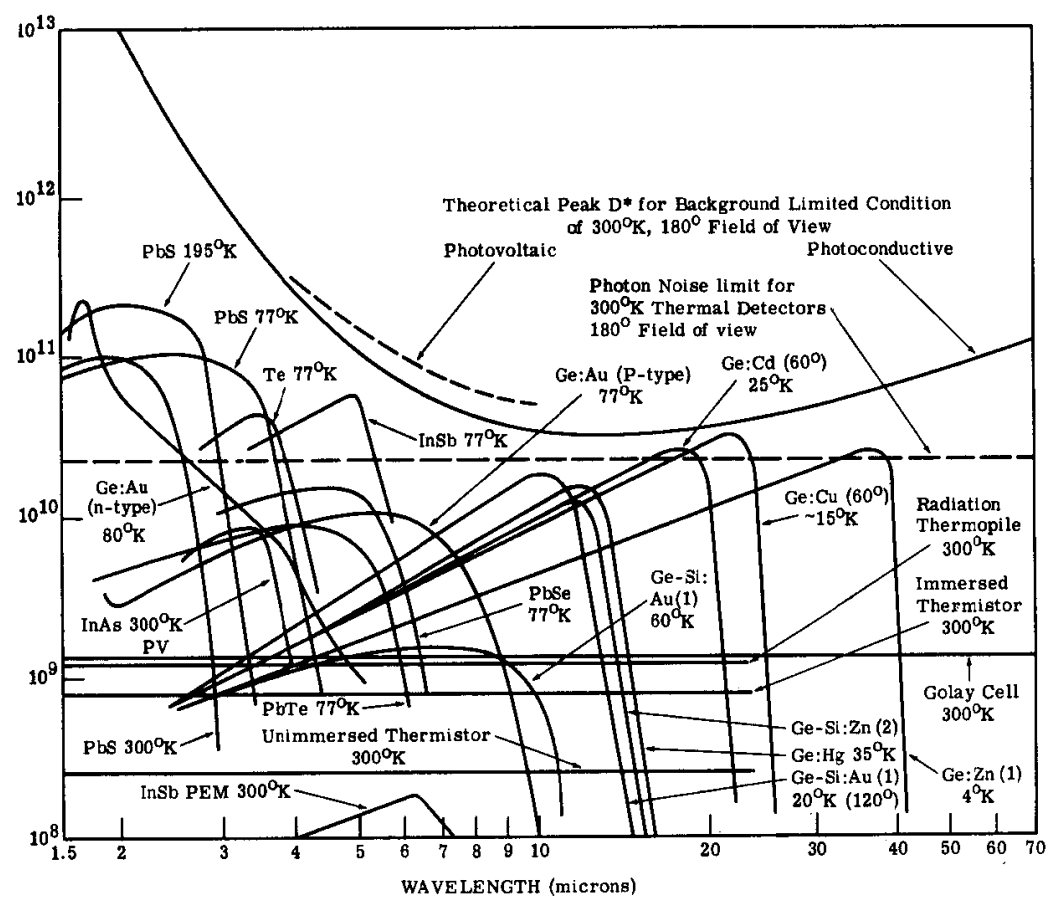

Figure 1. Detectivity of most infrared detectors.

The first example, a device built by the Barnes Engineering Company called the Thermograph $\mathrm{T}-1$, is the forerunner of the instrument now described as their Medical Thermograph. It can be seen by reference to the schematic shown in FIGURE 3 that the instrument incorporates a reference blackbody and a chopper which causes the detector to "see" the patient and the blackbody alternately. This technique is used fairly commonly in measuring instruments because the blackbody provides an almost constant calibration of the system. The total field of view is scanned by movement of the resolution field in a regular raster, programmed by movement of the entire system. Of course, the modern instrument uses a large flat mirror in front of the collecting optic to perform this function. FIgURE 4 shows another ancient and honorable device called the Reconofax built by Haller, Raymond, and Brown, Inc. (now HRB Singer). It has been used as an aerial reconnaissance device so that no attention has been given to an internal reference source; they have chosen to place the detector at the focus of the primary mirror. This is a question which often arises: which causes the optical efficiency to be smallest, the use of secondary optics with concomitant optical problems or the extra obscurations of a detector and its housing, preamplifier, and cables. Note that only one dimensional scanning is provided by the flat; the plane's movement is to furnish the second motion.

Two things should be noted about each of the above systems: they use a single mirror to scan and do the scanning either with or before the focusing or collecting mirror. This technique is called object-space scanning. It may be thought of as mechanically moving an optical pencil over the field, and therefore means that optical quality must remain good only over this pencil. 


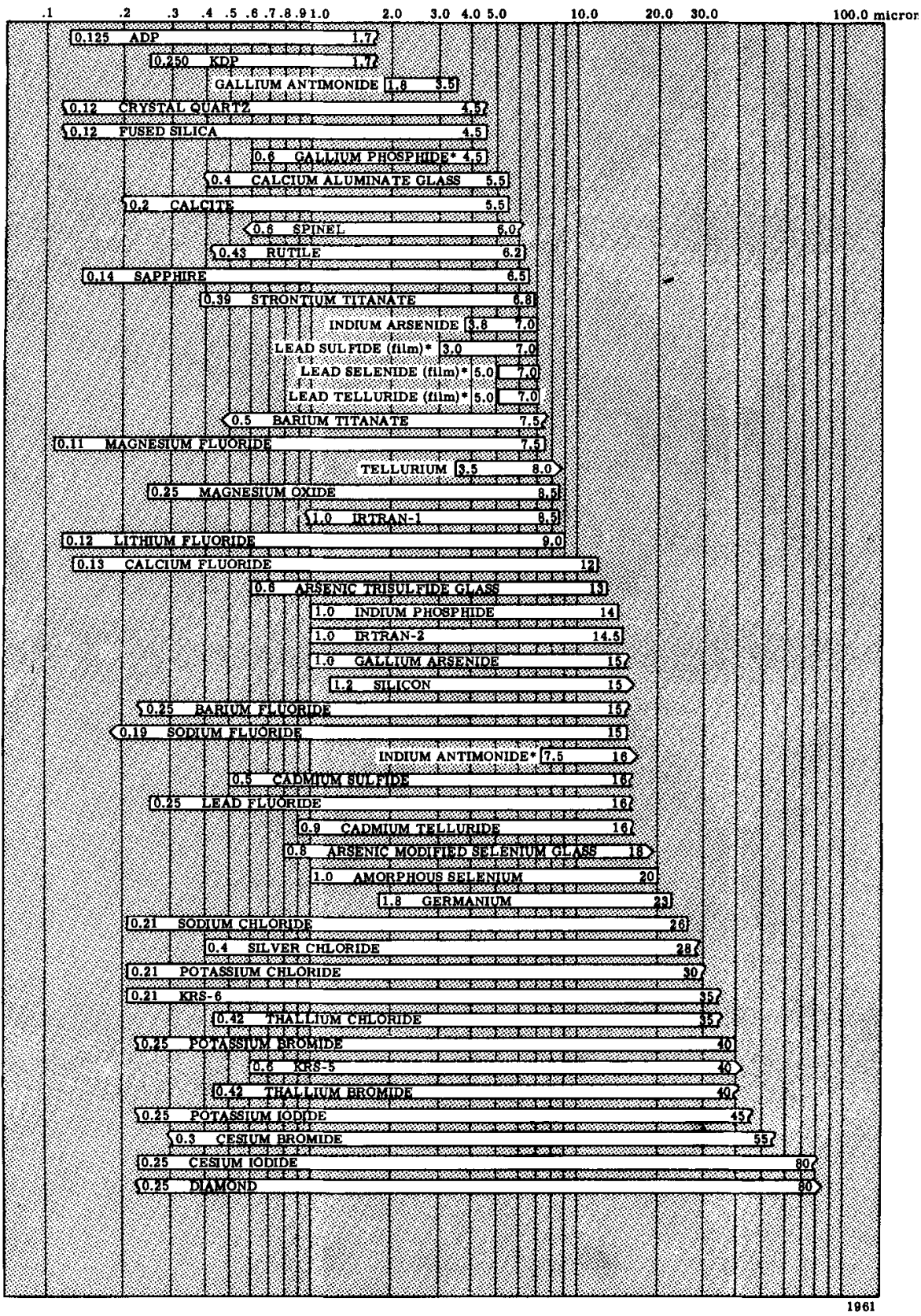

FIGURE 2. Transmission regions of most infrared optical materials. 


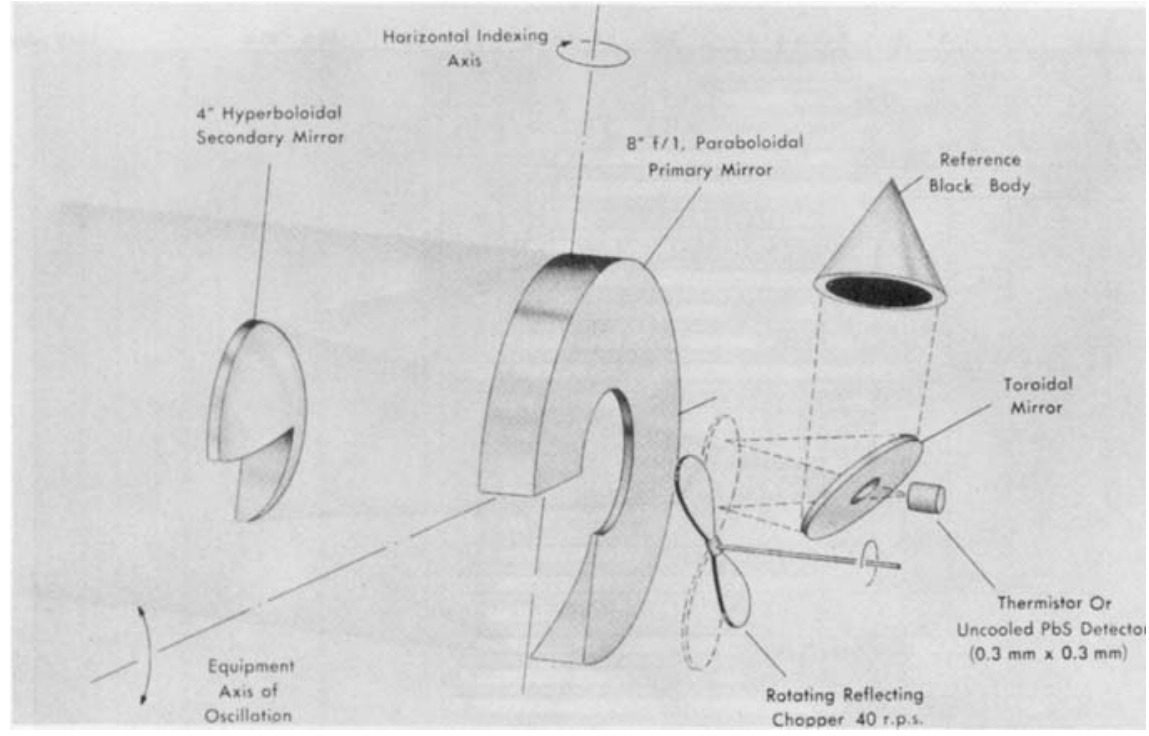

FIGURE 3. Barnes Thermograph T-1.
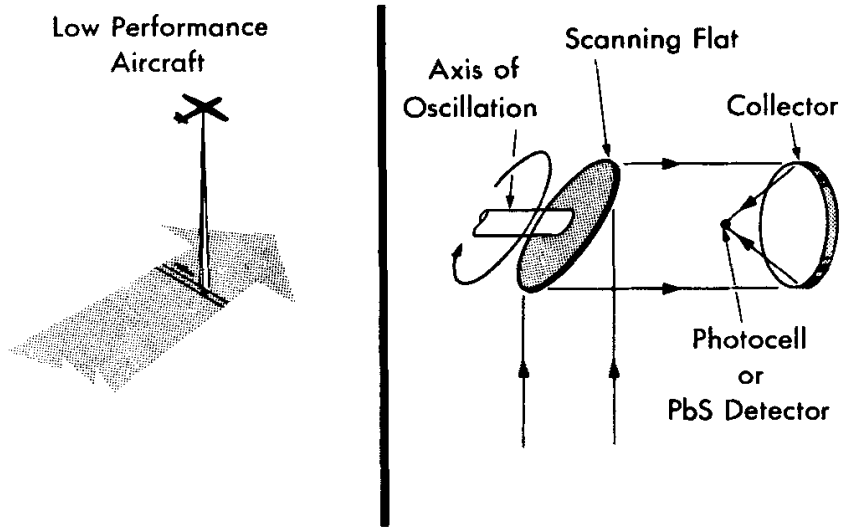

Figure 4. Reconfax schematic.

A more complicated system is shown in FIovre 5 where a collector and a folding secondary are used. Again there is a one-dimensional scan. In another simple example of devices of this type the detector is placed near the center of the folding flat, which in turn provides appropriate scanning action.

A scanning system built by Westinghouse is shown in FIGURE 6 . For total fields of view less than $180^{\circ}$ this is a more efficient system. It makes use of what is essentially a four-sided mirror, thereby scanning a field of about $90^{\circ}$ with 100 per cent duty cycle. Another way to do this is shown in FIGURE 7 . Note also that this device 
Wolfe: Infrared Imaging Devices

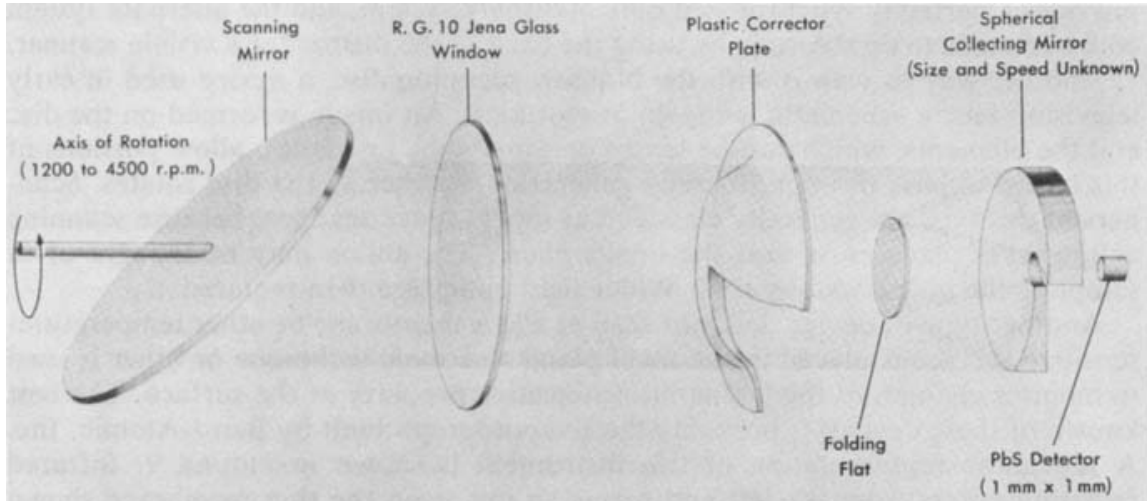

Figure 5. A more complicated Reconofax.

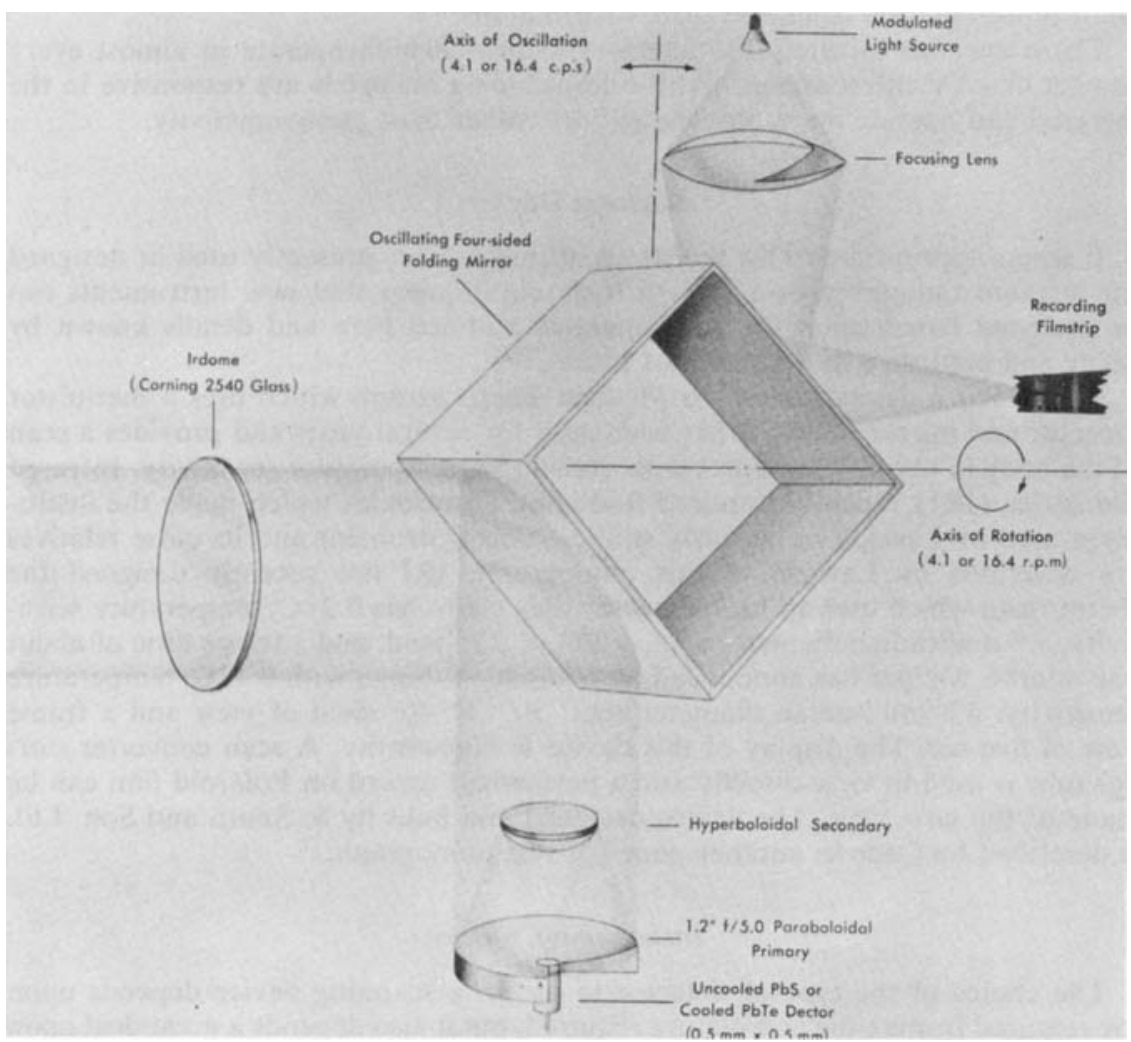

Figure 6. A Westinghouse scanner. 
provides a perfectly synchronized optical display system, and the alternate system could be made to do the same by using the back of the mirror for a visible scanner.

Another way to scan is with the Nipkow scanning disc, a device used in early television sets; a schematic is shown in FIGURE 8. An image is formed on the disc and the elements, which can be lenses or sometimes just holes, allow portions of this image to pass through, thereby generating a raster as the disc rotates. Scanners of this type are generally classified as image-space scanners because scanning action takes place in or near the image plane. The action may be thought of as sampling the image spot by spot. Wider field optics are then required.

Another type of device does not scan at all. A membrane or other temperaturesensitive surface is placed in the focal plane and some technique or other is used to monitor changes of the temperature-sensitive property of the surface. The best known of these devices is probably the Evaporograph built by Baird-Atomic, Inc. A schematic representation of this instrument is shown in FIGURE 9. Infrared radiation enters from the left and forms an image on the thin membrane shown in the center. The oil vapor in the right-hand chamber normally maintains a thin film of liquid oil on the membrane which the heat of the infrared image evaporates differentially, so that the film has different thickness over its area, depending upon the local infrared intensity. White light is viewed through the membrane which, because it is so thin, causes differential interference of this light. Thus the viewer sees different colors due to the different thicknesses. The viewer sees a color representation of temperature distributions.

There are also infrared TV tubes-vidicons-which operate in almost every respect like TV tubes except that the transducing materials are responsive in the infrared and operate on photoconductivity rather than photoemissivity.

\section{Current Devices}

It seems appropriate to list the status of instruments presently used or designed for infrared radiography -although it should be clear that new instruments can be designed based upon the fundamentals outlined here and details known by many and published in a number of places. ${ }^{1-5}$

Barnes has a device called the Medical Thermograph which uses a thermistor detector and mirror optics; it has been used for several years and provides a scan of the body in about 12 minutes with about $1^{\circ} \mathrm{K}$ temperature sensitivity. Infrared Industries (IRI) recently acquired Radiation Electronics which made the instrument used by Lawson in his early studies. This instrument and its close relatives are described by Lawson in this monograph. IRI has recently designed the thermoscan which uses an InSb detector they claim has $0.2^{\circ} \mathrm{C}$. temperature sensitivity, 3.5 milliradian diameter spot, a $20^{\circ} \times 20^{\circ}$ field, and a frame time of about one minute. Melpar has announced an equipment design with $0.1^{\circ} \mathrm{C}$. temperature sensitivity, 5.8 milliradian diameter spot, $30^{\circ} \times 40^{\circ}$ field of view and a frame time of five sec. The display of this device is noteworthy. A scan converter storage tube is used to view directly and a permanent record on Polaroid film can be made of the next scan. The device designed and built by S. Smith and Son, Ltd. is described by Cade in another paper of this monograph.

\section{Instrument Choices}

The choice of the type of detector to use in a scanning device depends upon the required frame time and pictire required, but it also depends a great deal upon expense and convenience. Thermistors, thermocouples, thermopiles and other 


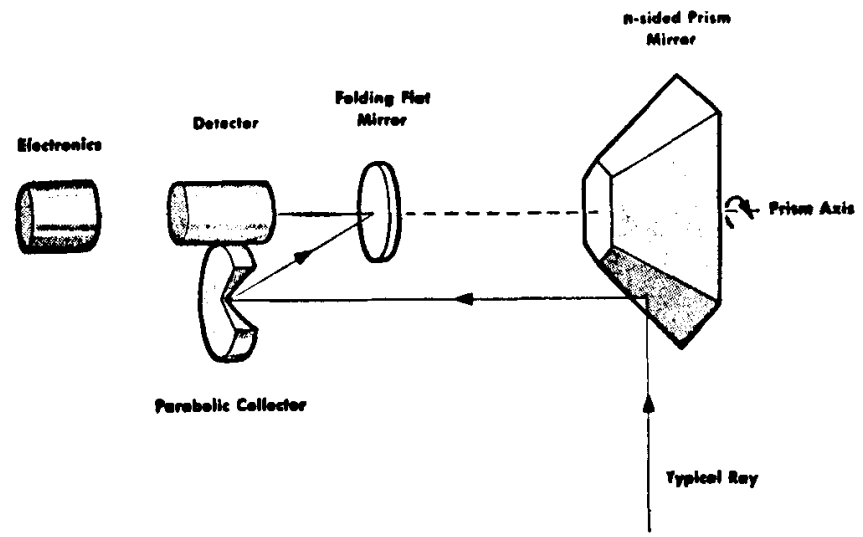

Figure 7. A wedge scanner

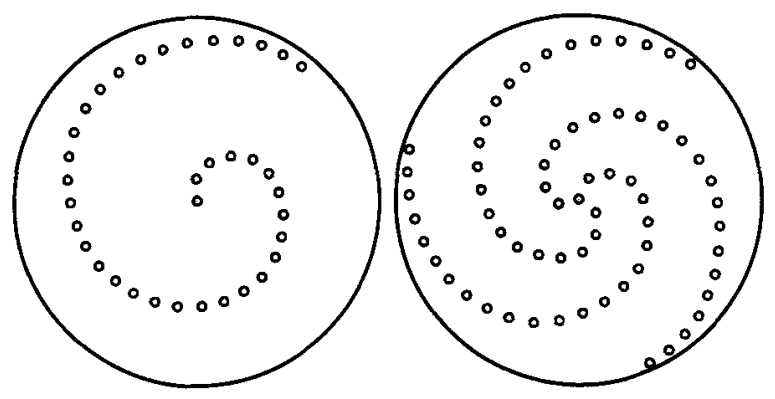

Figure 8. Nipkow discs.

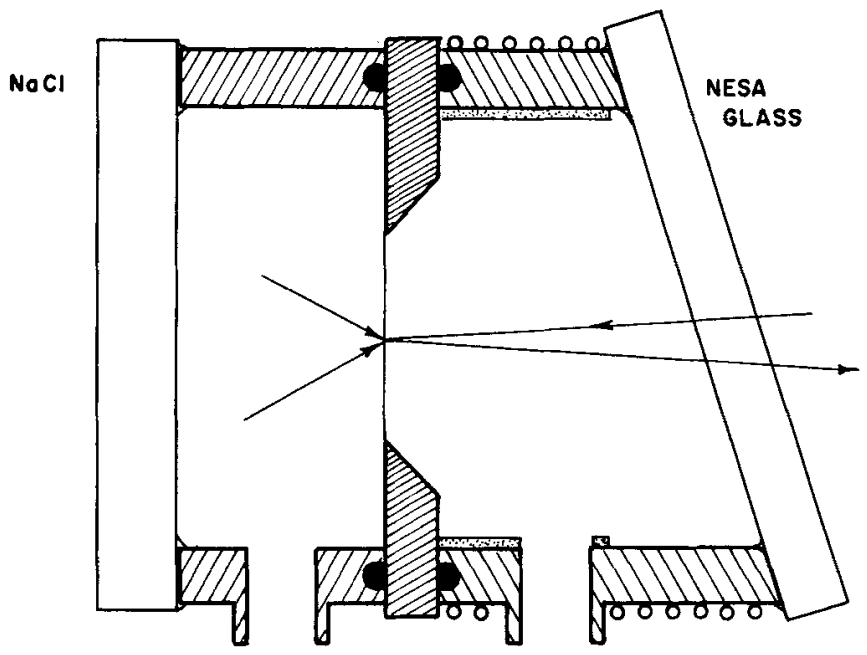

Figure 9. Baird-Atomic Evaporograph. 
thermal detectors, which have a constant sensitivity (to within a factor of two, no matter what the wavelength) have $D^{*}$ values of about $10^{9}$ and time constants of about one msec. Photodetectors (see figure 1) are about three orders of magnitude faster, two orders more sensitive, one order more expensive, definitely not flat (but relatively smooth) and require cooling.

The best spectral region of operation seems to be longer than seven $\mu$ in the large atmospheric transmission window. This is because the light in the examination room can give rise to spurious reflections. Tungsten lights are more likely to give appreciable radiation at longer wavelengths than are fluorescents, and radiation slide rule calculations show that 90 per cent of the light from a $2958^{\circ} \mathrm{K}$ tungsten bulb is below three $\mu$ : 99 per cent is below $7.5 \mu$. Only 10 per cent of the radiation from the human body lies below seven $\mu$. The emissivity of skin is probably very close to one and very nearly constant from 7-14 $\mu$ although it seems to show appreciable reflectivity at shorter wavelengths. The spectral region in question has good atmospheric transmission and it can also be shown that the position or wavelength of maximum radiation difference resulting from a temperature difference is given by

$$
\lambda_{\text {max }} \mathrm{T}=5,100 \mu^{\circ} \mathrm{K}
$$

This gives a $\lambda_{\max }$ for body temperature of $17 \mu$.

\section{An Optimum Instrument}

Because there are still so many undetermined variables, it is difficult to give any firm specifications about the optimum instrument. In fact, it may even be that several different devices will be used. It is possible at this time, however, to specify some things about a research instrument. It should use a fast photoconductive detector such as mercury-doped germanium to take advantage of its higher detectivity and much faster time constant. This will permit investigations of patient cooling effects, of the value of very high spatial and temperature resolution, and of the effects in different spectral regions; it will also provide greater experience with cooled systems, and a comparison of these results with more conventional instruments, thereby providing information on which engineering compromises can be made. The equipment should be all reflective, have provision for the use of a variety of filters, and possibly be a two-channel device so that measurements can be made almost simultaneously in two different spectral regions. The output should be put on tape so that optimum displays can be investigated.

\section{Conclusion}

Infrared radiography is one of the most exciting applications of infrared technology. While in its infancy it can benefit from the experience of the infrared experts who have spent years applying the theory and techniques to a variety of problems. There are some equipments available for clinical use, more on the drawing board, but there exists no ideal instrument. This can only be designed after additional experience has been gained by the clinician and research worker.

\section{Appendix A : Radiometric Fundamentals}

Infrared radiation like visible light is an arbitrarily specified part of electromagnetic radiation. The only physical difference between the two is the wavelength. Conceptually the physics underlying the nature of the radiation has profound depth and implications, but for the purposes of understanding infrared 
radiography it is only necessary to know a small portion of the ideas. The basic law derived by Max Planck in 1901, prescribes the power per unit of area per unit wavelength interval of a perfect emitter called a blackbody as

$$
W_{\lambda}=8 \pi c^{2} h \lambda^{-5}\left(e^{h c} / \lambda k T-1\right)^{-1}
$$

The quantity $W_{\lambda}$ is called the spectral radiant emittance. A somewhat simpler form is

$$
\mathrm{W}_{\lambda}=\mathrm{c}_{1} \lambda^{-5}\left(\mathrm{e}^{\left.\mathrm{c}_{2}{ }^{\lambda / \mathrm{T}}-1\right)^{-1}}\right.
$$

Here, all the constants have been collected into $c_{1}$ and $c_{2}$; it can be seen that the radiation in a narrow wavelength band $W_{\lambda}$ varies with the wavelength and with temperature. Clearly the total radiant emittance $W$ which comes from a perfect radiator is obtained by summing the radiation in every small spectral interval, that is by integrating $W_{\lambda}$ from zero to infinity; the radiant emittance in any spectral interval $W_{\Delta \lambda}$ is obtained by integrating the Planck equation over the proper limits.

$$
\mathrm{W}_{\Delta \lambda}=\int \begin{aligned}
& \lambda_{1} \\
& \lambda_{2}
\end{aligned} \mathrm{~W}_{\lambda} \mathrm{d}_{\lambda}, \quad \Delta \lambda=\lambda_{2}-\lambda_{1}
$$

The maximum of the blackbody is determined by the Wien Displacement Law.

$$
\lambda_{\max } \mathrm{T}=2,897 \mu^{\mathrm{o}} \mathrm{K}
$$

The calculations are usually difficult enough that special tables and computational aids are used. Fortunately, the total radiant power density $W$ is given simply:

$$
\mathrm{W}=\sigma \mathbf{T}^{4}
$$

Further, $W_{\Delta \lambda}$ can usually be written as $\sigma \mathrm{T}^{\mathrm{n}}$ where $\mathrm{n}$ is determined by the part of the curve being considered. In both cases $\sigma$ is the Stefan-Boltzmann constant, $T$ is the absolute temperature and $\mathrm{W}$ is usually stated in terms of watt $\mathrm{cm} .^{-2}$ or equivalent units like cal $\mathrm{sec}^{-1} \mathrm{~cm}^{2}$

These are the fundamental radiation equations for perfect radiators. Natural radiators are not perfect; their degree of perfection is characterized by a radiation efficiency factor called emissivity $\epsilon$. A perfect radiator has an emissivity of one. Thus, the Stefan-Boltzmann radiation equation must be rewritten:

$$
\mathrm{W}=\epsilon \sigma \mathrm{T}^{4}
$$

It is important to understand the essence of the radiometric measurement process. By the theory just presented it is clear that everything around is more or less luminous in the infrared; to coin a word, all objects are "radious." For instance, a blackboard in our classroom one meter on a side radiates a total of about six kilowatts! All objects radiate to each other, the net radiation passing from warmer to cooler objects consonant with the third law of thermodynamics.

A radiometric device is basically a system which collects all the radiation from a weli-defined field of view, focuses it onto an element which transduces this radiation level to a visible or electrical recording. Such a simple radiometer would record zero output only if everything in the field of view has a temperature exactly equal to the internal temperature of the radiometer. It is obvious then that the radiometer conceived here will only record the difference between the radiation levels in two different fields of view. The practical equation for radiometric use therefore deals with temperature differences. A frequently used technique is to have the radiometer view the unknown field and a known source alternately; the known source is usually a good approximation to a perfect radiator and may be considered perfect $(\epsilon=1)$. Then the output signal is related to the difference between these two radiant emittances

$$
\begin{gathered}
\mathrm{W}_{\text {signal }}=\mathrm{W}_{\text {unknown }}=\mathrm{W}_{\text {standard }}=\mathrm{W}_{\mathrm{u}}-\mathrm{W}_{\mathrm{k}} \\
=\sigma\left(\epsilon_{\mathrm{u}} \mathrm{T}_{\mathrm{u}}{ }^{4}-\mathrm{T}_{\mathrm{k}}{ }^{4}\right)
\end{gathered}
$$

where the subscript $u$ represents the unknown and $k$ the known standard. 
The temperature $T_{s}$ is measured; $\sigma$ is known, and $W_{s}$ is measured. The unknown temperature can only be obtained if the unknown emissivity is measured somehow. Even if two objects which have the same unknown emissivity and different temperatures are measured, there is still not enough information for determination of temperature. These equations cannot be solved simultaneously in any way to obtain values of $T_{11}$ without a knowledge of $\epsilon_{11}$.

If the radiometer discussed above views first one portion of the object (patient), then the standard and then another portion of the object and so on, a series of different outputs will be obtained. In obtaining differences in radiation from the different parts of the patient, the level of the standard (if it remains constant) drops from the calculations:

$$
\begin{gathered}
\Delta \mathrm{W}_{\mathrm{sig}}=\left(\mathrm{W}_{\mathrm{u}_{1}}-\mathrm{W}_{\mathrm{k}_{1}}\right)-\left(\mathrm{W}_{\mathrm{u}_{2}}-\mathrm{W}_{\mathrm{k}_{2}}\right) \\
\mathrm{W}_{\mathrm{k}_{1}}=\mathrm{W}_{\mathrm{k}_{2}} \\
\Delta \mathrm{W}_{\mathrm{sig}}=\mathrm{W}_{\mathrm{u}_{1}}-\mathrm{W}_{\mathrm{u}_{2}}=\Delta \mathrm{W}
\end{gathered}
$$

Thus, output signal differences represent patient radiation differences. The possible causes for such changes can be determined without great difficulty in two ways. Each depends upon a careful statement of the radiation equation for the situation. The body radiates according to a $T^{n}$ law, where $n$ is determined by the spectral region. The body emits according to its emissivity and reflects radiation from the examining room walls according to its reflectivity $\rho$ and the temperature of the walls $T_{0}$

$$
W=\sigma\left(\epsilon T^{n}+\rho T_{o}{ }^{n}\right)
$$

To a good approximation $\rho$ is equal to $(1-\epsilon)$; thus

$$
\mathrm{W}=\sigma\left[\epsilon \mathrm{T}^{\mathrm{n}}+(1-\epsilon) \mathrm{T}_{n}{ }^{\mathrm{n}}\right]
$$

It is reasonable to assume that $T_{0}$ does not change; $\sigma$ is a constant; therefore $W$ can change only if $\epsilon$ or $T$ (or both) change. Thus

$$
\mathrm{W}+\Delta \mathrm{W}=\sigma\left\{(\epsilon+\Delta \epsilon)(\mathrm{T}+\Delta \mathrm{T})^{n}+[1-(\epsilon+\Delta \epsilon)] \mathrm{T}_{0}{ }^{n}\right\}
$$

The difference $\Delta W$ is obtained by subtracting $W$ from $W+\Delta W$.

By a laborious algebraic process and by assuming that values of $\Delta T$ and $\Delta \epsilon$ raised to the second power are negligible, one can show that

$$
\Delta \mathrm{W}=\sigma\left(4 \epsilon \mathrm{T}^{3} \Delta \mathrm{T}+\mathrm{T}^{4} \Delta \epsilon-\mathrm{T}_{0}^{4} \Delta \epsilon\right)
$$

(This is more easily done by differential calculus and verification that second order differences are negligible.) By simple substitution it can be shown for temperatures of about $300^{\circ} \mathrm{K}$, a change of 0.02 in emissivity is equivalent to a change of $1^{\circ} \mathrm{K}$ in temperature. This equation also shows that the examining room should be at about body temperature for changes to be due to temperature alone. Of course, lower room temperatures increase the contrast. This just emphasizes the need for an accurate knowledge of skin emissivity in sickness and in health.

The logic presented above is based on the measurement of actual temperature differences. An analysis providing relative temperature differences is obtained by dividing $\Delta \mathrm{W}$ by $\mathrm{W}$. Then

$$
\frac{\Delta W}{W}=4 \frac{\Delta T}{T}+\frac{\Delta \epsilon}{\epsilon}
$$

Substitution of $\epsilon=1, \Delta E=0.02$, and $\mathrm{T}=300^{\circ} \mathrm{K}$ shows that a relative error of 0.02 in $\epsilon$ is equivalent to a relative error of 0.013 in $T$. The results agree, as of course they must. 


\section{Appendix B: Derivation of the Design Equation}

The equation written in the text is

$$
\mathrm{NETD}=\left(\begin{array}{c}
\pi \\
\bar{\sigma}
\end{array}\right)\left(\frac{1}{\epsilon \mathrm{T}^{3}}\right)\left(\frac{\Omega}{\omega^{2}}\right)\left(\frac{1}{\eta \mathbf{A}_{\mathrm{c}}}\right)\left(\frac{\sqrt{\mathrm{A}_{\mathrm{d}}}}{\mathrm{D}^{*}}\right)\left(\frac{1}{\mathrm{t}}\right)
$$

The terms are also defined there. The derivation begins with a consideration of the power radiated from the patient's surface.

The power per unit area is written $W$ and called radiant emittance. The power per unit area per unit solid angle is called radiance and written $\mathbf{N}$. (A solid angle is a two-dimensional angle - an area divided by a distance squared.) Thus $P_{c}$ the power on an optical collector is the product of $\mathbf{N}$ and the source area $\mathbf{A}_{\mathbf{s}}$ and the solid angle intercepted by the collector:

$$
\mathbf{P}_{\mathrm{c}}=\mathrm{NA}_{\mathrm{s}} \omega_{\mathrm{sc}}=\frac{\mathrm{NA} \mathrm{A}_{\mathrm{s}} \mathbf{A}_{\mathrm{c}}}{\mathrm{r}^{2}}
$$

The power density at the collector, the irradiance $H$, is given as

$$
\mathrm{H}=\frac{\mathbf{P}_{\mathrm{c}}}{\mathrm{A}}=\frac{\mathrm{NA}}{\mathrm{r}_{\mathrm{s}}}
$$

The noise equivalent irradiance is the irradiance change which produces an output signal just equal to that which internal noises produce. Thus

$$
\mathrm{NEH}=\frac{\mathrm{NEP}}{\mathrm{A}_{\mathrm{c}} \eta}
$$

where $\eta$ represents optical efficiency.

This relationship assumes that all the power on the collector gets to the detector. The noise equivalent power of the detector is the power required to give an output equal to the noise. Thus dividing NEP by $A_{c}$ gives $N E H$.

The difference in irradiance due to temperature differences on the patient's skin is

$$
\Delta H=\frac{N A_{s}}{r^{2}}=\frac{W}{\pi} \frac{A_{s}}{r^{2}}=\frac{\epsilon \sigma T^{3} \Delta T A_{s}}{\pi r^{2}}
$$

The noise equivalent temperature difference is given by

$$
\mathrm{NETD}=\frac{\mathrm{NEH}}{\Delta \mathrm{H} / \Delta \mathrm{T}}=\left(\frac{\mathrm{NEP}}{\eta \mathrm{A}_{\mathrm{c}}}\right)\left(\frac{\pi \mathrm{r}^{2}}{\epsilon \sigma \mathrm{T}^{3} \mathrm{~A}_{\mathrm{s}}}\right)
$$

Detector performance is usually specified in terms of $D^{*}$ (FIGURE 1 ) where $D^{*}$ is given by

Then

$$
D^{*}=\frac{\sqrt{A_{d} \Delta f}}{\text { NEP }}
$$

$$
\mathrm{NETD}=\left(\frac{\pi}{\sigma}\right)\left(\frac{1}{\epsilon \mathrm{T}^{3}}\right)\left(\frac{\mathrm{r}^{2}}{\mathrm{~A}_{\mathrm{s}}}\right)\left(\frac{1}{\eta \mathrm{A}_{\mathrm{c}}}\right)\left(\frac{\sqrt{\mathrm{A}_{\mathrm{d}}}}{\mathrm{D}^{*}}\right) \sqrt{\Delta \mathrm{f}}
$$

The bandwidth is related to the time constant $\tau$ according to information theory:

$$
\Delta \mathrm{f}=\frac{1}{2 \tau}
$$

If the system must spend two time constants on each small resolution element, then

$$
\frac{2 \tau}{t}=\frac{A_{s}}{A_{t}}
$$


where $t$ is the total frame time

$A_{s}$ is the skin resolution element size

$A_{t}$ is the total area to be scanned

Thus

$$
\Delta f=\frac{1}{2 \tau}=\frac{A_{t}}{A_{s}} t=\frac{\left(A_{t} / r^{2}\right) t}{\left(A_{s} / r^{2}\right.}=\frac{\Omega t}{\omega}
$$

When this quantity is substituted for $\Delta \mathrm{f}$, the desired equation is obtained.

\section{References}

1. M. Holter, S. Nudelman, G. Suits, W. Wolfe \& G. Zissis. 1962. Fundamentals of Infrared Technology. The Macmilian Co. New York, N. Y.

2. P. Kruse, L. McGlauchlin \& R. McQuistan. 1962. Elements of Infrared Technology. John Wiley \& Sons, Inc. New York, N. Y.

3. Proc. IRE. 1959. 47: 1413-1700.

4. J. Jamieson, R. McFee, G. Plass, R. Grube \& R. Richards. 1963. Infrared Physics and Engineering. McGraw-Hill Book Co., New York, N. Y.

5. J. King, T. Limperis, J. Morgan, F. Polcyn \& W. Wolfe. 1963. Intl. Sci. Tech. : 26-37. 